\title{
Bone morphogenetic protein-9 promotes the differentiation of mouse spleen macrophages into osteoclasts via the ALK1 receptor and ERK 1/2 pathways in vitro
}

\author{
HAI $^{1 *}{ }^{1 *}$, DAHANG ZHAO ${ }^{1,2^{*}}$, SHENGJING WANG ${ }^{1}$, JING DING $^{1}$ and LI ZHAO ${ }^{1}$ \\ ${ }^{1}$ Department of Pediatric Orthopaedics, Xin-Hua Hospital Affiliated to Shanghai Jiao Tong University School of Medicine, \\ Shanghai 200092; ${ }^{2}$ The Third Department of Orthopedics, The First Affiliated Hospital of Harbin Medical University, \\ Harbin, Heilongjiang 150001, P.R. China
}

Received August 26, 2015; Accepted September 12, 2016

DOI: $10.3892 / \mathrm{mmr} .2016 .5803$

\begin{abstract}
It has been confirmed that bone morphogenetic protein-9 (BMP-9) promotes the differentiation of osteoblasts. However, the ways in which BMP-9 exerts its effects on the differentiation of osteoclasts and bone resorption remain to be elucidated. The present study was designed to investigate the roles and the molecular mechanism of BMP-9 on the proliferation and differentiation of osteoclast precursors in vitro. Mouse spleen macrophages (RAW 264.7 cells) were cultured in the presence of receptor activator for nuclear factor-kb ligand (RANKL) in vitro. Following treatment with different concentrations of BMP-9, a number of parameters were quantitatively monitored. Cell proliferation was determined using an MTT assay. The expression levels of cell BMP receptor-IA (BMPR-IA), BMPR-IB, BMPR-II and anaplastic lymphoma kinase 1 (ALK1) receptor were detected by ELISA, the small mothers against decapentaplegic pathway, extracellular signal-regulated kinase (ERK)1/2 pathways and markers of osteoclast differentiation were detected by western blotting. The results showed that
\end{abstract}

Correspondence to: $\mathrm{Dr} \mathrm{Li}$ Zhao, Department of Pediatric Orthopaedics, Xin-Hua Hospital Affiliated to Shanghai Jiao Tong University School of Medicine, 1665 Kongjiang Road, Shanghai 200092, P.R. China

E-mail: orthzl@126.com

*Contributed equally

Abbreviations: BMP-9, bone morphogenetic protein-9; RANKL, receptor activator for nuclear factor- $\mathrm{bb}$ ligand; BMPR, bone morphogenetic protein receptor; ALK1, anaplastic lymphoma kinase 1; ERK, extracellular signal-regulated kinase; CTR, calcitonin receptor; TRAP, tartrate-resistant acid phosphatase; ELISA, enzyme-linked immunosorbent assay

Key words: bone morphogenetic protein-9, mice spleen macrophages, osteoclast formation, anaplastic lymphoma kinase 1 receptor, extracellular signal-regulated kinase $1 / 2$ signaling pathways treatment with BMP-9 alone promoted mouse spleen macrophage proliferation, and the differentiation into osteoclasts occurred only in the presence of RANK. The promoting effect of BMP-9 on cell proliferation and osteoclast differentiation occurred in dose-dependent manner. In addition, BMP-9 significantly upregulated the expression of the ALK1 receptor and inhibited the ERK1/2 pathway. The inhibition of the ERK1/2 pathways was ameliorated by transfection with small interfering (si)RNA ALK1. The effect of BMP-9 on osteoclast differentiation was reduced by transfection with siRNA ALK1, however, the effect was enhanced by the ERK1/2 pathway inhibitor, U0126. The results of the present study demonstrated that BMP-9 promoted the osteoclast differentiation of osteoclast precursors via binding to the ALK1 receptor on the cell surface, and inhibiting the ERK1/2 signaling pathways in the cell.

\section{Introduction}

Skeletal modeling is a physiological process combining bone formation with bone resorption, where osteoclasts, derived from hematopoietic mononuclear macrophages, are involved in bone resorption. Under the stimulation of receptor activator for nuclear factor-kb ligand (RANKL) and macrophage colony stimulating factor, mononuclear macrophages fuse to form osteoclasts and express osteoclast markers, including calcitonin receptor (CTR) and RANK, with the activation of tartrate-resistant acid phosphatase (TRAP) for bone resorption $(1,2)$. As previously reported, osteoclast differentiation is regulated by several stimulators, including several types of growth factors present in the bone microenvironment (3). It is of clinical significance to investigate the regulatory mechanism of osteoclast differentiation, which may contribute to the development of novel drugs for inhibiting bone resorption.

Bone morphogenetic proteins (BMPs) are multi-functional growth factors in the transforming growth factor- $\beta$ superfamily, which is associated with numerous molecular cascades and signaling pathways (4). At present, 18 different BMP-homologous molecules have been identified, and have been shown to induce effects via the small mothers against decapentaplegic (Smad) and mitogen-activated protein kinase 
pathways through binding to different types of receptors, including BMP receptor (BMPR)-I, BMPR-II and anaplastic lymphoma kinases (ALKs) $(4,5)$. A number of previous studies have confirmed the effect of BMPs on osteogenesis, including the differentiation of preosteoblasts and bone matrix synthesis (5-8). The effects of BMPs on osteoclastogenesis and bone resorption have also attracted scientific interest, however, the effects remain to be fully elucidated (1). It has been suggested that BMP-9 may promote the differentiation of osteoblasts, however, the effects of BMP-9 on the differentiation of osteoclasts and bone resorption remain to be fully elucidated $(7,9)$. In the present study, mouse spleen macrophages (RAW 264.7 cells) were cultured as osteoclast precursors to investigate the roles of BMP-9 on osteoclast proliferation and differentiation, and to examine the signaling mechanism involved. The aim of the present study was to determine the potential impact of BMP-9 on bone resorption in bone homeostasis.

\section{Materials and methods}

Cell culture. Mouse spleen macrophages were purchased from the Cell Bank of Chinese Academy of Sciences (Shanghai, China) for use as osteoclast precursors. These cells were cultured in Dulbecco's minimum essential medium (Gibco; Thermo Fisher Scientific, Inc., Waltham, MA, USA) containing $10 \%$ fetal bovine serum (FBS; Thermo Fisher Scientific, Inc.) at $37^{\circ} \mathrm{C}$ in a humidified incubator containing $5 \% \mathrm{CO}_{2}$, and the medium was replaced every 3 days. The exponentially growing cells were seeded at a density of $2 \times 10^{4}$ cells/well in 48 -well plates for the examinations of cell proliferation, TRAP-positive cell staining and immunofluorescence staining, or at a density of $2 \times 10^{5}$ cells/well in six-well plates for enzyme-linked immunosorbent assay (ELISA) and western blot analysis. Soluble recombinant human (rh)RANKL (100 ng/ml; PeproTech, Inc., Rocky Hill, NJ, USA) was added to stimulate osteoclastogenesis of the preosteoclasts. After $24 \mathrm{~h}$ of incubation, different concentrations $(0-150 \mathrm{ng} / \mathrm{ml})$ of rhBMPs (R\&D Systems, Inc., Minneapolis, MN, USA) were added to the cultures. The medium, BMPs and RANKL were replaced every 3 days. Triplicate cell cultures were performed for the gene experiment, and quadruple cell cultures were performed for the other experiments. Experiments were repeated twice to confirm the results.

Cell proliferation assay. An MTT assay was performed to evaluate cell viability, as previously described (2). Cells $\left(8 \times 10^{3}\right)$ were seeded onto 96 -well plates $24 \mathrm{~h}$ prior to treatment with ALK1-siRNA for $48 \mathrm{~h}$ using Lipofectamine ${ }^{\circledR} 2000$ (Invitrogen; Thermo Fisher Scientific, Inc.). After 48 h, $15 \mu l$ (5 mg/ml) MTT (Sigma-Aldrich; Merck Millipore, Darmstadt, Germany) was added to each well, and the cells were incubated for a further $4 \mathrm{~h}$ at $37^{\circ} \mathrm{C}$. Subsequently, $150 \mu \mathrm{l}$ dimethyl sulfoxide was added and the mixtures were agitated for $10-15$ min to fully dissolve the crystals. Absorbance (A) was measured at $570 \mathrm{~nm}$ using a Tecan microplate reader (Männedorf, Switzerland). Cell viability was calculated as the percentage change in A570 between the control and treated cells.

TRAP staining of cells. After $72 \mathrm{~h}$, the cells were fixed with 4\% paraformaldehyde for $10 \mathrm{~min}$, washed with PBS, and then incubated in TRAP staining ( $0.05 \mathrm{M}$ acetate buffer, $0.03 \mathrm{M}$ sodium tartrate, $100 \mathrm{~g} / \mathrm{ml}$ naphthol AS-MX phosphate, $0.01 \%$ Triton X-100 and $0.3 \mathrm{mg} / \mathrm{ml}$ Fast Red Violet LB stain) for 10 mins at $37^{\circ} \mathrm{C}$. A phase contrast microscope was used to observe and calculate the number of osteoclasts.

ELISA. The protein expression of BMPR-IA, BMPR-IB, BMPR-II, ALK1 receptors and CTR on the cell surface were detected using ELISA (Biomedical Technologies, Inc., Stoughton, MA, USA) according to the kit instructions. The results were standardized by the quality of glyceraldehyde-3-phosphate dehydrogenase (GAPDH).

Immunofluorescence detection. The cells were fixed with 4\% paraformaldehyde and blocked with 5\% BSA, and were then incubated overnight at $4^{\circ} \mathrm{C}$ with the following primary antibody: Polyclonal goat anti-mouse ALK1 immunoglobulin (Ig) G (1:100; cat. no. 770-MA; R\&D Systems, Inc., Minneapolis, MN, USA), quenched with glycine $(0.1 \mathrm{M})$ for $1 \mathrm{~h}$, incubated with secondary antibodies (polyclonal donkey anti-goat IgG; 1:200; cat. no. NL003; R\&D Systems, Inc.) for $2 \mathrm{~h}$ at room temperature, and then washed three times with PBS. The cells were then counterstained with DAPI (Vector Laboratories Canada, Inc., Burlington, ON, Canada), and the slides mounted in PBS-glycerol 50\% (v/v). The detection of fluorescence intensity was evaluated using fluorescence microscopy.

Western blot analysis. At the end of the culture period, the cells were washed with cold PBS, and lysed in the presence of protease inhibitors. Protein concentrations were determined using the Bio-Rad protein assay kit (Bio-Rad Laboratories, Inc., Hercules, CA, USA). Total proteins (50 $\mu \mathrm{g}$ ) were separated by $10 \%$ SDS-PAGE, and transferred onto a PVDF membrane (EMD Millipore, Billerica, MA, USA). The membrane was blocked with 5\% BSA in Tris-buffered saline Tween-20 for $1 \mathrm{~h}$ at room temperature. For visualization, the blots were probed with antibodies against Smad2 (1:1,000 dilution; cat. no. 3103), phosphorylated (p)-Smad2 (1:1,000 dilution; cat. no. 3104), Erk1/2 (1:500 dilution; cat. no. 4696), p-Erk1/2 (1:500 dilution; cat. no. 9101) and GAPDH (1:1,000 dilution; cat. no. 97166) (all Cell Signaling Technology, Inc., Danvers, MA, USA) overnight at $4^{\circ} \mathrm{C}$. Horseradish peroxidase-conjugated goat anti-rabbit (cat. no. 7074) and horse anti-mouse (cat. no. 7076) secondary antibodies (1:1,000; Cell Signaling Technology, Inc.) were used to enable detection using a chemiluminescent system at room temperature. The band densities were quantified using a Bio-Rad Versa Doc imaging system (Bio-Rad Laboratories, Inc.), and normalized to the GAPDH band as an internal control for each group.

RNA inactivation. The HP custom siRNA kit (cat. no. 1027423) was obtained from Qiagen (Mississauga, ON, Canada); the negative control (NC) sequence was included. A total of $48 \mathrm{~h}$ prior to stimulation, the differentiated cells were transfected either with a mouse ALK1 siRNA (NM_001277255.1, 5'-GCAGGAAATCTCACCACAT-3', 3'-AUGUGGUGAGAU UUCCUGCTT-5'; $1 \mathrm{ng} / \mathrm{ml}$; Qiagen) or with an NC siRNA (5'-GCAAATATCACCCAGGCAT-3', 3'-AUGCCUGGGUGA UAUUUGCTT-5'; $1 \mathrm{ng} / \mathrm{ml}$; Qiagen) in a solution containing Lipofectamine $^{\circledR} 2000$ transfection reagent in serum-free 
A

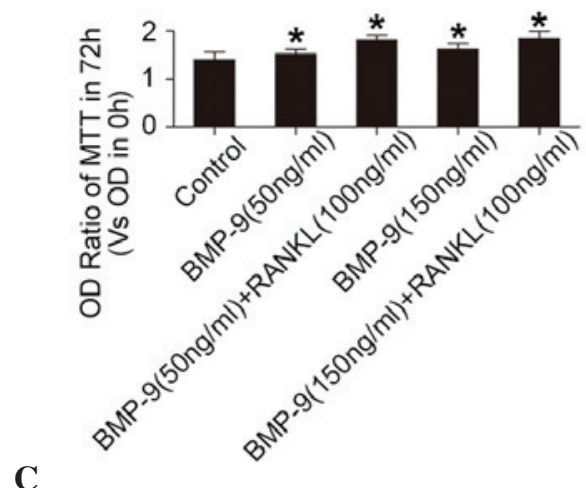

B

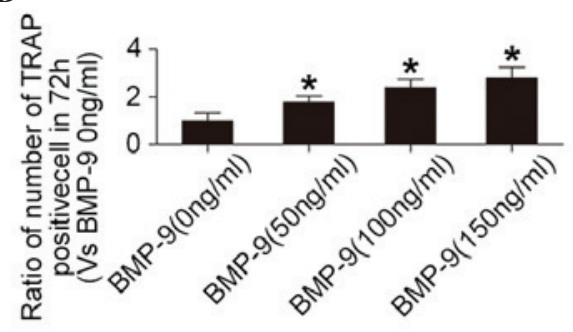

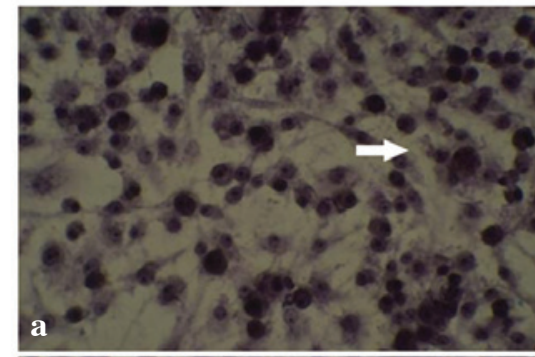

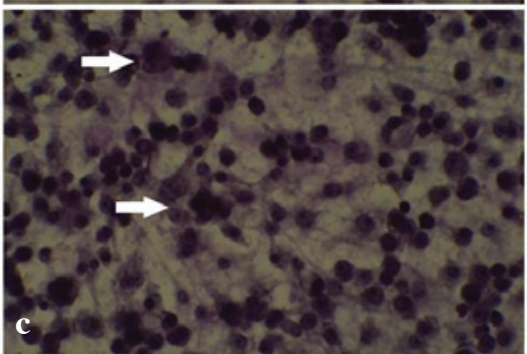

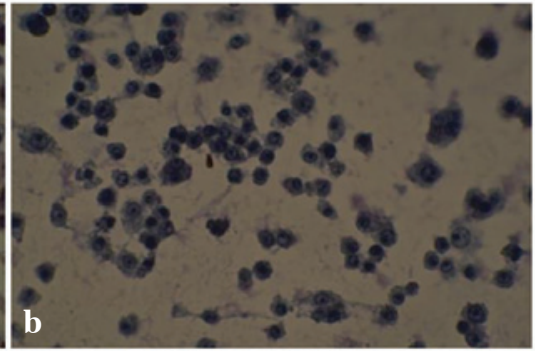

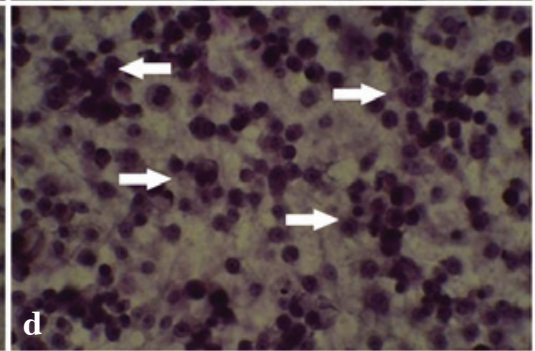

D

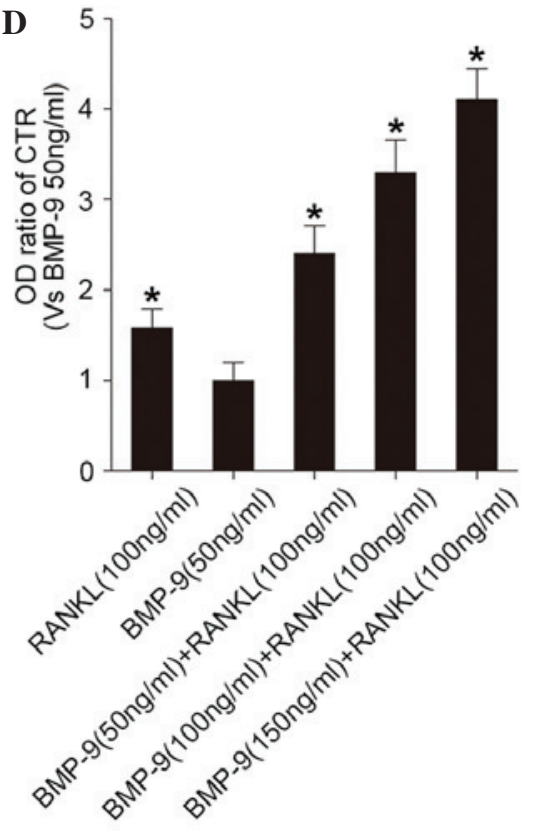

Figure 1. Effects of BMP-9 on osteoclast proliferation and differentiation. (A) Proliferation of cells in different treatment groups cultured for $72 \mathrm{~h}$, determined using an MTT assay. OD ratio $=\mathrm{OD}(0 \mathrm{~h}) / \mathrm{OD}(72 \mathrm{~h}) .{ }^{*} \mathrm{P}<0.01$, vs. control group. (B) Percentage of TRAP-positive cells in different treatment groups cultured for $72 \mathrm{~h}$. "P<0.01, vs. BMP-9 (0 ng/ml) group. (C) TRAP staining of cells treated with (a) $100 \mathrm{ng} / \mathrm{ml}$ RANKL without BMP-9, (b) $50 \mathrm{ng} / \mathrm{ml}$ BMP-9 without RANKL, (c) $50 \mathrm{ng} / \mathrm{ml}$ BMP-9 with RANKL (100 ng/ml), (d) $150 \mathrm{ng} / \mathrm{ml}$ BMP-9 with RANKL (100 ng/ml). A phase contrast microscope was used (magnification, x20). TRAP-positive cells are indicated by white arrows. (D) Expression of CTR, assessed using an enzyme-linked immunosorbent assay, following treatment with different concentrations of BMP-9 with or without RANKL. "P<0.01, vs. BMP-9 (50 ng/ml) group. BMP-9, bone morphogenetic protein-9; RANKL, receptor activator for nuclear factor-kb ligand; CTR, calcitonin receptor; OD, optical density; TRAP, tartrate-resistant acid phosphatase.

Opti-MEM. The cells were incubated at $37^{\circ} \mathrm{C}$ for $2 \mathrm{~h}$ with gentle stirring. The transfection efficiency was evaluated using fluorescence microscopy in one well of the culture containing a control green fluorescent protein siRNA, and the subsequent downregulation of ALK1 was assessed using western blotting.

Statistical analysis. Data are expressed as the mean \pm standard deviation of three or six independent experiments, and the statistical significance was determined using a paired Student's $t$-test or analysis of variance. Statistical analyses were conducted using SPSS 19.0 software (SPSS, Inc., Chicagi, IL, USA) and graphs were generated using GraphPad Prism 5.0 (GraphPad Software, Inc., La Jolla, CA, USA). $\mathrm{P}<0.01$ was considered to indicate a statistically significant difference.

\section{Results}

Effects of BMP-9 on osteoclast proliferation and differentiation. The MTT assay showed that RANKL $(100 \mathrm{ng} / \mathrm{ml})$ and BMP-9 (50-150 $\mathrm{ng} / \mathrm{ml})$ alone promoted cell proliferation (Fig. 1A). The TRAP staining of the cells showed that RANKL (100 ng/ml) stimulated the activation of TRAP in the preosteoclasts (Fig. 1B and Ca). However, BMP-9 $(50-150 \mathrm{ng} / \mathrm{ml})$ failed to induce the activation of TRAP in the cells without the presence of RANKL (Fig. 1Cb). In the presence of RANKL $(100 \mathrm{ng} / \mathrm{ml})$, the percentage of TRAP-positive cells was significantly elevated by BMP-9 (50-150 $\mathrm{ng} / \mathrm{ml}$ ) in a dose-dependent manner (Fig. 1B and Cc and d). The effect of BMP-9 on the activation of TRAP in the cells was not associated with its effect on cell proliferation. In addition, the expression of the CTR marker in the cell, detected using ELISA showed a similar trend (Fig. 1D). This suggested that BMP-9 promoted RANKL-induced osteoclast differentiation in a dose-dependent manner at certain concentrations $(50-150 \mathrm{ng} / \mathrm{ml})$.

Effects of BMP-9 on the expression of BMP receptors. The BMPR-IA, BMPR-IB, BMPR-II and ALK1 receptors were detected in the cells induced by BMP-9 $(100 \mathrm{ng} / \mathrm{ml})$ using ELISA. Among these receptors, the ALK1 receptor showed the highest level of upregulation (Fig. 2A). According to 
A

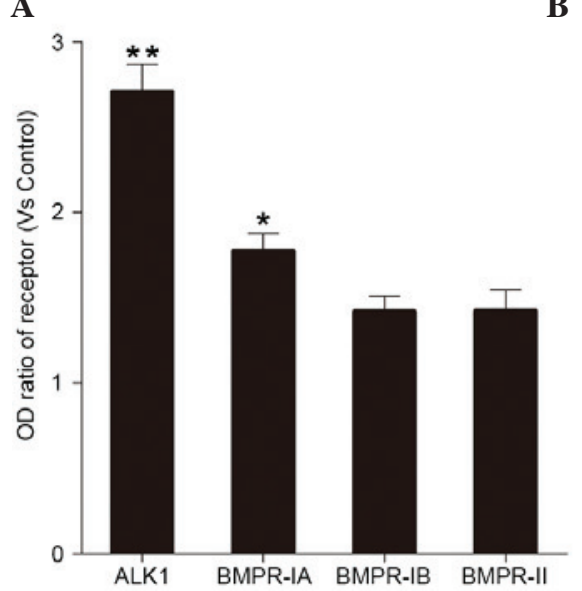

B

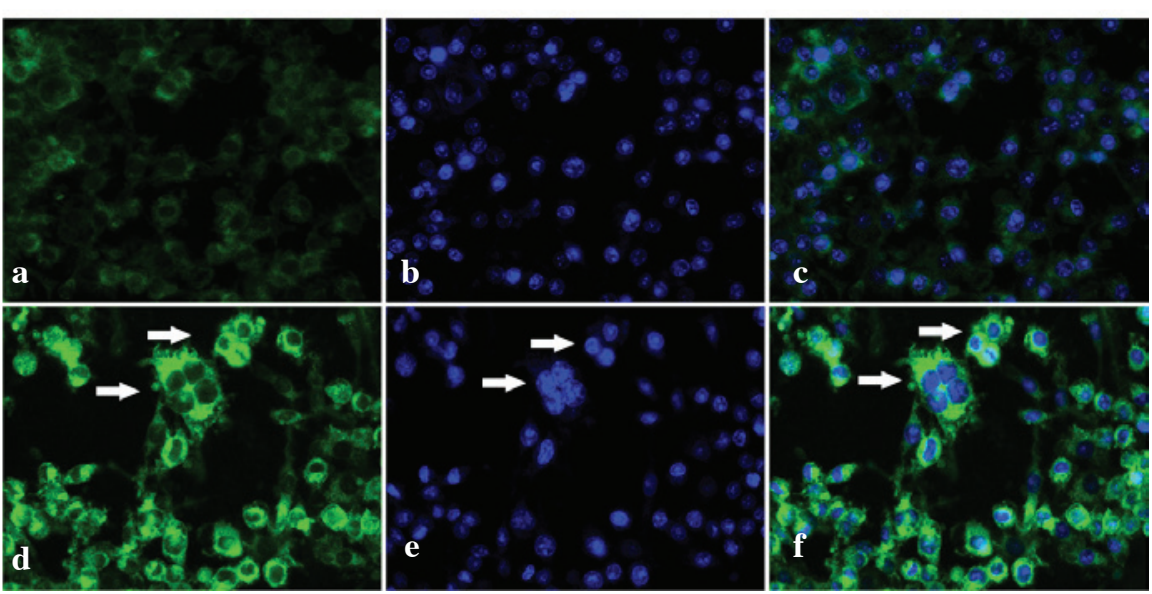

Figure 2. Effects of BMP-9 on the expression of BMP receptors. (A) Expression of BMPR-IA, BMPR-IB, BMPR-II and ALK1 receptors in cells treated with BMP-9 (100 ng/ml) using ELISA. * P $<0.01$ and "P<0.05, vs. other receptors. (B) Immunofluorescence of (a) ALKI receptor, (b) counterstaining with DAPI and (c) the two stains merged in cells without BMP-9 treatment. Immunofluorescence of (d) ALKI receptor (e) counterstaining with DAPI and (f) the two stains merged in cells treated with BMP-9 $(100 \mathrm{ng} / \mathrm{ml})$. Positive expression of ALK1 receptor is indicated by white arrows. BMP-9, bone morphogenetic protein-9; BMPR, BMP receptor; ALK1, anaplastic lymphoma kinase 1; OD, optical density.

previous reports (10-12), BMP-9 preferentially bind to its ALK1 receptor. Therefore, in the present study, the ALK1 receptor was selected and its expression on the cell surface was confirmed using immunofluorescence (Fig. 2Ba-f).

Effects of BMP-9 and the ALK1 receptor on the induction of the cell signal transduction pathway. The Smad2 and non-Smad EPK1/2 signaling pathways are the most common pathways in cells induced by BMP-9 $(4,5)$. The results of the western blot analysis showed that the phosphorylation of ERK1/2 (p-ERK1/2) was decreased in the cells following the induction of BMP-9, and was significantly reduced at 60 and 120 min, respectively (Fig. 3A and B). However, no change was observed in the phosphorylation of Smad2 during induction for 0-120 min (Fig. 3C and D). These results showed that BMP-9 inhibited the ERK1/2 pathway in the osteoclast precursor. Furthermore, the inhibited phosphorylation of ERK1/2 was reduced in the cells pre-transfected with siRNA-ALK1 (Fig. 3E and F). This suggested that BMP-9 inhibited the phosphorylation of ERK1/2 through the ALK1 receptor in the cells.

Effects of the ALK1 receptor and ERKI/2 pathways on BMP-9-induced differentiation of osteoclasts. In the presence of RANKL, BMP-9 (100 ng/ml) induced the activation of TRAP in the cells Fig. 4Aa. The percentage of TRAP-positive cells was reduced in the cells pre-transfected with siRNA-ALK1 Fig. 4Ab), but increased in the cells exposed to U0126 $(1,000 \mathrm{nmol} / \mathrm{l})$, an inhibitor of the ERK1/2 pathway (Fig. 4Ac). These results suggested that the promotion of BMP-9 on osteoclast differentiation was reduced by inhibition of the ALK1 receptor, but enhanced by inhibition of the ERK1/2 pathways. In addition, the protein expression of CTR in the cells, determined using ELISA, showed similar results (Fig. 4B). Therefore, BMP-9 inhibited the intracellular ERK1/2 pathways through mediation of the ALK1 receptor on the surface of macrophagocytes, further promoting the differentiation into osteoclasts.

\section{Discussion}

The present in vitro study showed that BMP receptors, including BMPR-IA, BMPR-IB, BMPR-II and ALK1, were detected in mouse spleen macrophages, and that BMP-9 promoted the proliferation and osteoclast differentiation of the cells in the presence of RANKL. The possible mechanism underlying the promotion of differentiation by BMP-9 may be that BMP-9 inhibited the intracellular EPK1/2 pathways through binding to ALK1 receptors on the cell surface.

Previous studies have confirmed that BMPs, including BMP-2, 4 and 7, not only promote osteogenisis differentiation, but are also important in regulating osteoclast differentiation $(6,13)$. Due to its importance in osteogenesis, BMP-9 is considered to be a growth factor offering significant potential in clinical practice. However, there are few reports on the roles of BMP-9 in osteoclast differentiation and bone resorption. The effects of BMP-9 on osteoclast differentiation were confirmed by the results of the present study, which also offered novel clues to its possible mechanism. A previous study by Fong et al (14) suggested that BMP-9 did not promote osteoclast differentiation in human mononuclear macrophages, however, it enhanced bone resorption by significantly inhibiting the apoptosis of mononuclear macrophages in the presence of RANKL. The findings of the present study showed that BMP-9 promoted the proliferation of mouse spleen mononuclear macrophages, and enhanced osteoclast differentiation only in the presence of RANKL. These findings show the direct effects of BMP-9 on osteoclast precursors and bone resorption. However, the effect of BMP-9 on cell differentiation was not associated with its effect on cell proliferation, which suggested that BMP-9 may have different effects on mononuclear macrophages from different sources. In mononuclear macrophages in the mouse spleen, a study by Zheng et al (15) demonstrated that the BMP2/7 heterodimer promoted proliferation and osteoclast differentiation in a dose-dependent manner in the presence of RANKL in vitro. These findings suggest that BMP factors have effects 
A

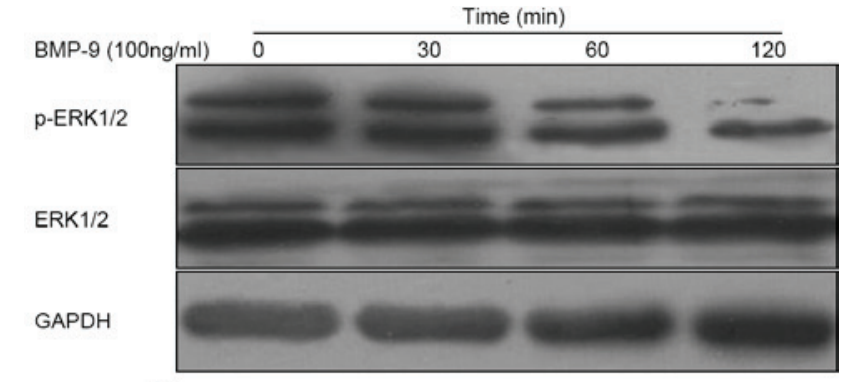

B

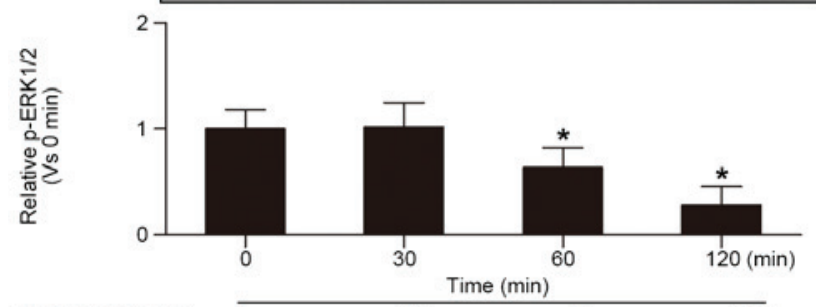

C

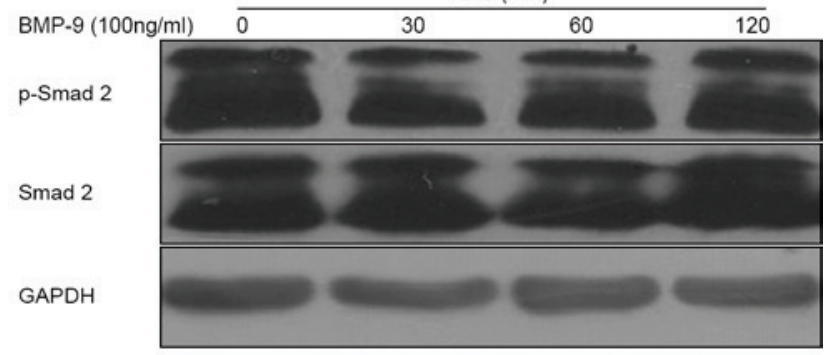

D

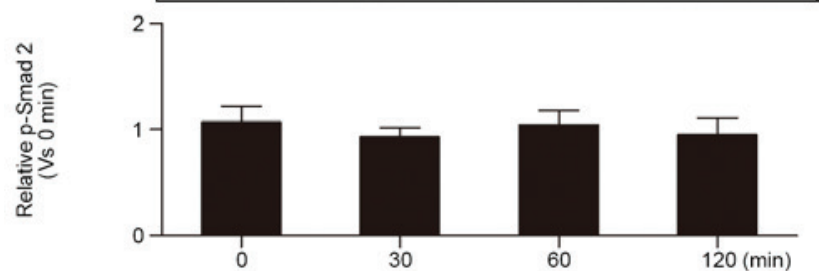

$\mathbf{E}$

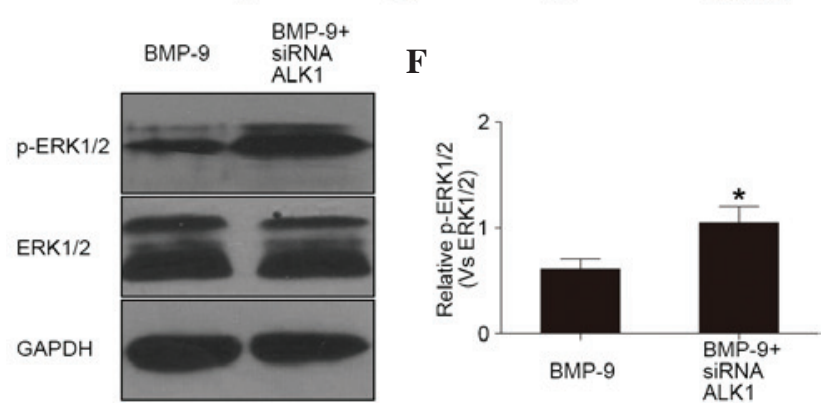

Figure 3. Effects of BMP-9 and ALK1 receptor on the cell signal transduction pathway. (A) Western blot of the phosphorylation of ERK1/2 in cells treated with BMP-9 $(100 \mathrm{ng} / \mathrm{ml})$ and (B) quantification ("P<0.01, vs. 0 min). (C) Western blot of the phosphorylation of Smad2 in cells treated with BMP-9 (100 ng/ml) and (D) quantification (P>0.01, vs. 0 min). (E) Western blot of the phosphorylation of ERK1/2 in cells pre-treated with siRNA-ALK1 and (F) quantification ("P<0.01, vs. BMP-9). ERK1/2, extracellular signal-regulated kinase 1/2; p-ERK1/2, phosphorylated ERK1/2; Smad 2, small mothers against decapentaplegic 2; p-Smad2, phosphorylated Smad 2; BMP-9, bone morphogenetic protein-9; ALK1, anaplastic lymphoma kinase 1; siRNA, small interfering RNA.

A

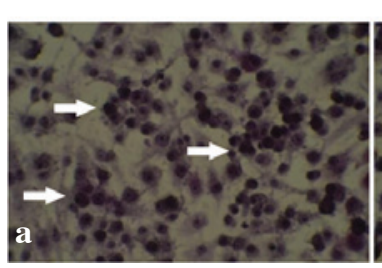

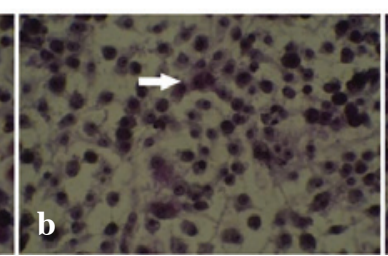

B

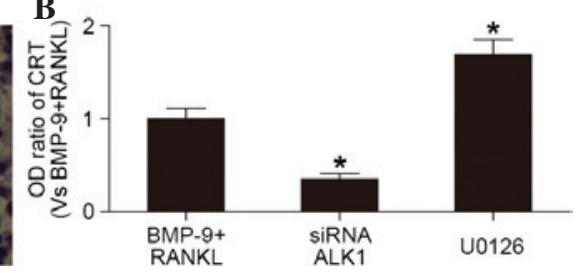

Figure 4. Effects of the ALK1 receptor and ERK1/2 pathways on BMP-9-induced osteoclast differentiation (A) TRAP staining of cells (a) induced by BMP-9 $(100 \mathrm{ng} / \mathrm{ml})$ and RANKL (100 ng/ml), and (b) pre-transfected with siRNA-ALK1 or (c) cultured with U0126 (1,000 nmol/l) prior to BMP-9+RANKL. (Phase contrast microscope; magnification, x20). White arrows indicate TRAP-positive cells. (B) Protein expression of CTR, determined using an enzyme-linked immunosorbent assay, in cells treated with BMP-9 $(100 \mathrm{ng} / \mathrm{ml})$ and RANKL $(100 \mathrm{ng} / \mathrm{ml})$, transfectecd with siRNA-ALK1 or treated with U0126 (1,000 nmol/l). "P<0.01, vs. BMP-9+RANKL group. BMP-9, bone morphogenetic protein-9; ALK1, anaplastic lymphoma kinase 1; RANKL, receptor activator for nuclear factor- $\kappa b$ ligand; CTR, calcitonin receptor; siRNA, small interfering RNA; OD, optical density; TRAP, tartrate-resistant acid phosphatase. 
on osteoclast differentiation with diversity according to the different osteoclast precursors.

Various types of BMP receptors, including BMPR-IA, BMPR-IB, BMPR-II and ALK1,2, have been found in osteoclast precursors from different sources. BMP-9 is the physiological ligand of ALK1,2 in various cells $(12,16)$, and it has also been reported that BMP-9 binds to its BMPR-II receptor in osteoclast precursors (12). In the present study, the expression levels of BMPR-IA, BMPR-IB, BMPR-II and ALK1 receptors were detected in the cells induced by BMP-9. Among these receptors, the expression of ALK1 was upregulated significantly. Using an siRNA inactivation assay, the ALK1 receptor was found to be important in the effect of osteoclast differentiation by BMP-9. It is important to acknowledge that the involvement of the other receptors on the effects of BMP-9 cannot be excluded. The Smad2 and Smad-independent ERK1/2 pathways are the primary signaling pathways in cells induced by BMP-9 (16-18). The findings from the present study showed that BMP-9 inhibited the phosphorylation of ERK1/2 pathways. However, a study by Fong et al (14) indicated that BMP-9 promoted the ERK1/2 pathways, suggesting that the binding of BMP-9 to different receptors may have different effects.

There were two limitations to the present study: i) As BMP-9 is similar to other BMPs, it may bind to different receptors and activate, or inactivate, different signaling pathways in different cell types. In the present study, only the ALK1 receptor and EPK1/2 signaling pathways were investigated, as investigated in previous studies $(4,5,7,16,19,20)$, and their effects on the impact of BMP-9 were confirmed. However, whether another receptor or signaling pathway is involved in this signaling mechanism remains to be elucidated. Therefore, considering the complexity of the signaling mechanism by BMPs, further investigations are required on other receptors or signaling pathways; ii) As in vitro results are not necessarily consistent with the effects of BMP-9 in vivo, there is a requirement to perform further in vivo investigations.

In conclusion, the presents study confirmed that BMP-9 promoted the proliferation and differentiation of osteoclast precursors in the presence of RANKL, which involved the ALK1 receptor and ERK1/2 pathways. These findings expand on current understanding of the effects of BMPs on the regulation of osteoclast differentiation and bone resorption, and provide experimental evidence for further in vivo investigations.

\section{Acknowledgements}

This study was supported by the Science Foundation of Shanghai Science and Technology Commission (grant no. 11ZR1423900).

\section{References}

1. Boyle WJ, Simonet WS and Lacey DL: Osteoclast differentiation and activation. Nature 423: 337-342, 2003.
2. Parfitt AM, Mundy GR, Roodman GD, Hughes DE and Boyce BF: A new model for the regulation of bone resorption, with particular reference to the effects of bisphosphonates. J Bone Miner Res 11: 150-159, 1996.

3. Graves L III and Jilka RL: Comparison of bone and parathyroid hormone as stimulators of osteoclast development and activity in calvarial cell cultures from normal and osteopetrotic (mi/mi) mice. J Cell Physiol 145: 102-109, 1990.

4. Bragdon B, Moseychuk O, Saldanha S, King D, Julian J and Nohe A: Bone morphogenetic proteins: A critical review. Cell Signal 23: 609-620, 2011.

5. Schmierer B and Hill CS: TGFbeta-SMAD signal transduction: Molecular specificity and functional flexibility. Nat Rev Mol Cell Biol 8: 970-982, 2007.

6. Lavery K, Swain P, Falb D and Alaoui-Ismaili MH: BMP-2/4 and BMP-6/7 differentially utilize cell surface receptors to induce osteoblastic differentiation of human bone marrow-derived mesenchymal stem cells. J Biol Chem 283: 20948-20958, 2008.

7. Luu HH, Song WX, Luo X, Manning D, Luo J, Deng ZL, Sharff KA, Montag AG, Haydon RC and He TC: Distinct roles of bone morphogenetic proteins in osteogenic differentiation of mesenchymal stem cells. J Orthop Res 25: 665-677, 2007.

8. Senta H, Park H, Bergeron E, Drevelle O, Fong D, Leblanc E, Cabana F, Roux S, Grenier G and Faucheux N: Cell responses to bone morphogenetic proteins and peptides derived from them: Biomedical applications and limitations. Cytokine Growth Factor Rev 20: 213-222, 2009.

9. Bergeron E,Leblanc E, Drevelle O, Giguère R, Beauvais S, Grenier G and Faucheux N: The evaluation of ectopic bone formation induced by delivery systems for bone morphogenetic protein- 9 or its derived peptide. Tissue Eng Part A 18: 342-352, 2012.

10. Bergeron E, Senta H, Mailloux A, Park H, Lord E and Faucheux N: Murine preosteoblast differentiation induced by a peptide derived from bone morphogenetic proteins-9. Tissue Eng Part A 15: 3341-3349, 2009.

11. Cunha SI and Pietras K: ALK1 as an emerging target for antiangiogenic therapy of cancer. Blood 117: 6999-7006, 2011.

12. Scharpfenecker M, van Dinther M, Liu Z, van Bezooijen RL, Zhao Q, Pukac L, Löwik CW and ten Dijke P: BMP-9 signals via ALK1 and inhibits bFGF-induced endothelial cell proliferation and VEGF-stimulated angiogenesis. J Cell Sci 120: 964-972, 2007.

13. David L, Feige JJ and Bailly S: Emerging role of bone morphogenetic proteins in angiogenesis. Cytokine Growth Factor Rev 20: 203-212, 2009.

14. Fong D, Bisson M, Laberge G, McManus S, Grenier G, Faucheux N and Roux S: Bone morphogenetic protein-9 activates Smad and ERK pathways and supports human osteoclast function and survival in vitro. Cell Signal 25: 717-728, 2013.

15. Zheng Y, Wang L, Zhang X, Zhang X, Gu Z and Wu G: BMP2/7 heterodimer can modulate all cellular events of the in vitro RANKL-mediated osteoclastogenesis, respectively, in different dose patterns. Tissue Eng Part A 18: 621-630, 2012.

16. Broege A, Pham L, Jensen ED, Emery A, Huang TH, Stemig M, Beppu H, Petryk A, O'Connor M, Mansky K and Gopalakrishnan R: Bone morphogenetic proteins signal via SMAD and mitogen-activated protein (MAP) kinase pathways at distinct times during osteoclastogenesis. J Biol Chem 288: 37230-37240, 2013.

17. Itoh K, Udagawa N, Katagiri T, Iemura S, Ueno N, Yasuda $H$, Higashio K, Quinn JM, Gillespie MT, Martin TJ, et al: Bone morphogenetic protein 2 stimulates osteoclast differentiation and survival supported by receptor activator of nuclear factor-kappaB ligand. Endocrinology 142: 3656-3662, 2001.

18. Jensen ED, Pham L, Billington CJ Jr, Espe K, Carlson AE, Westendorf JJ, Petryk A, Gopalakrishnan R and Mansky K: Bone morphogenic protein 2 directly enhances differentiation of murine osteoclast precursors. J Cell Biochem 109: 672-682, 2010.

19. Kaneko H, Arakawa T, Mano H, Kaneda T, Ogasawara A, Nakagawa M, Toyama Y, Yabe Y, Kumegawa M and Hakeda Y: Direct stimulation of osteoclastic bone resorption by bone morphogenetic protein (BMP)-2 and expression of BMP receptors in mature osteoclasts. Bone 27: 479-486, 2000.

20. Xie F, Liu W, Feng F, Li X, Yang L, Lv D, Qin X, Li L and Chen L: A static pressure sensitive receptor APJ promote H9c2 cardiomyocyte hypertrophy via PI3K-autophagy pathway. Acta Biochim Biophys Sin (Shanghai) 46: 699-708, 2014. 